Physics

Physics Research Publications

\title{
Resonant electron spin-flip Raman scattering in CdTe and the diluted magnetic semiconductor $\mathrm{Cd} 1-\mathrm{xVxTe}$
}
S. Tsoi
I. Miotkowski
S. Rodriguez
A. K. Ramdas
H. Alawadhi
T. M. Pekarek 


\title{
Resonant electron spin-flip Raman scattering in CdTe and the diluted magnetic semiconductor $\mathrm{Cd}_{1-x} \mathrm{~V}_{x} \mathrm{Te}$
}

\author{
S. Tsoi, I. Miotkowski, S. Rodriguez, and A. K. Ramdas \\ Department of Physics, Purdue University, West Lafayette, Indiana 47907, USA \\ H. Alawadhi \\ University of Sharjah, UAE \\ T. M. Pekarek \\ Department of Chemistry and Physics, University of North Florida, Jacksonville, Florida 32224, USA
}

(Received 17 September 2003; published 29 January 2004)

\begin{abstract}
Resonant enhancement enables the discovery and delineation of spin-flip Raman scattering (SFRS) from free or donor-bound electrons in diluted magnetic semiconductors containing $3 d$ transition-metal ions at doping concentrations. In such studies, the intrinsic $g$ factor of the host, CdTe in the present case, has to be accounted for accurately. The SFRS in CdTe yields the conduction band electron $g$ factor of $-1.676 \pm 0.007$ and displays two resonance peaks mediated by free and donor-bound excitons, respectively. Excitonic signature in modulated reflectivity signals the successful formation of $\mathrm{Cd}_{1-x} \mathrm{~V}_{x} \mathrm{Te}$ as an alloy, whereas magnetization measurements show vanadium ions incorporated as $\mathrm{V}^{2+}$ with $x \sim 4 \times 10^{-4}$. SFRS of $\mathrm{Cd}_{1-x} \mathrm{~V}_{x} \mathrm{Te}$ displays vanadium related SFRS shifts of a sign opposite to that of the host. This indicates a ferromagnetic $s-d$ exchange interaction between the $s$-like conduction electrons and the $3 d$ shell of $\mathrm{V}^{2+}$ in $\mathrm{Cd}_{1-x} \mathrm{~V}_{x} \mathrm{Te}$; from the linear dependence of the $s-d$ exchange energy as a function of magnetization $\alpha N_{0}$, the $s-d$ exchange constant is deduced to be $285 \pm 8 \mathrm{meV}$.
\end{abstract}

DOI: 10.1103/PhysRevB.69.035209

PACS number(s): 78.30.Fs, 78.20.Ls, 71.70.Gm, 71.70.Ej

\section{INTRODUCTION}

Diluted magnetic semiconductors (DMS's) are ternary compounds in which the cation sites are partially and randomly occupied by magnetic ions, for example, $3 d$ transition-metal ions (TMI's). ${ }^{1} \mathrm{Cd}_{1-x} \mathrm{Mn}_{x} \mathrm{Te}, \mathrm{Ga}_{1-x} \mathrm{Mn}_{x} \mathrm{As}$, and $\mathrm{Pb}_{1-x} \mathrm{Mn}_{x} \mathrm{Te}$ are examples of II-VI, III-V, and IV-VI DMS ternaries, ${ }^{1-3}$ respectively. Thanks to the exchange interactions between the TMI's as well as those between TMI's and band carriers, DMS's display striking magnetic and magneto-optic phenomena. To date, the most thoroughly investigated DMS's are Mn-, Co- and Fe-based II-VI DMS's in view of the large concentration of the magnetic ions which can be incorporated in bulk crystals; in CdTe, for example, $\mathrm{Mn}, \mathrm{Co}$, and $\mathrm{Fe}$ can be introduced to concentration levels of $70 \%, 2 \%$, and $\sim 4 \%$, respectively. Since the $3 d$ TMI's in the II-VI DMS's are isoelectronic with the cations, except for the distinctly magnetic $3 d$ cores, they offer an outstanding opportunity to investigate magnetic phenomena with a variety of experimental techniques and theoretical models. Currently, there is considerable interest in the study of III-V based DMS's with $\mathrm{Mn}$ (e.g., $\left.\mathrm{Ga}_{1-x} \mathrm{Mn}_{x} \mathrm{As}\right) .^{2}$ In these materials $\mathrm{Mn}^{2+}$ ion, replacing substitutionally the group III cation, is simultaneously a magnetic constituent and a single acceptor. This scenario sets the stage for solid-state electronics in which both the charge and the spin of carriers-holes in this case-are exploited, these ternaries then being referred to as spintronic semiconductors. ${ }^{4}$

While Mn-, Co-, and Fe based II-VI ternaries have attracted the most attention, the DMS's with other $3 d$ TMI's, viz $\mathrm{Sc}, \mathrm{Ti}, \mathrm{V}, \mathrm{Cr}, \mathrm{Ni}$, and $\mathrm{Cu}$ have been studied only to a limited extent, because only doping concentrations of these TMI's can been incorporated. However, the distinct type of magnetism displayed by each TMI is a strong motivation for the study of these DMS's, provided a suitable experimental technique is exploited. Additional interest in $\mathrm{Sc}, \mathrm{Ti}, \mathrm{V}$, and $\mathrm{Cr}$ based DMS's stems from theoretical predictions ${ }^{5}$ and preliminary experimental indications $s^{6}$ that the $p-d$ exchange interaction in DMS's with TMI's with less than a half-filled $3 d$ shell is ferromagnetic, in striking contrast to its antiferromagnetic character in those with $\mathrm{Mn}^{2+}, \mathrm{Co}^{2+}$, and $\mathrm{Fe}^{2+}$. It is in this context that we have undertaken the growth and magnetic and spectroscopic investigations of $\mathrm{Cd}_{1-x} \mathrm{~V}_{x} \mathrm{Te}$.

Spin-flip Raman scattering (SFRS) from free electrons or those bound to donors in the effective mass ground state is a powerful tool in the study of the magnetic phenomena displayed by DMS's. ${ }^{7}$ The SFRS shift in these cases equals the Zeeman splitting of the conduction band and thus yields the magnitude of the $s-d$ exchange interaction between the $d$ electrons of the TMI's and the $s$-like electrons of the $\Gamma_{6}$ conduction band. In contrast, other magnetooptic techniques, such as Faraday rotation, magnetoreflectance, and magnetoluminescence, involve the combined Zeeman splitting of the conduction and valence bands. In addition, the polarization selection rules of SFRS provide unique signatures of the underlying exchange interaction, whether it is ferromagnetic or antiferromagnetic. Another advantage of SFRS is its superior sensitivity and precision, especially desirable at low TMI concentrations, for which excitonic Zeeman splittings are small and the signatures observed in reflectivity and absorption tend to overlap. Further enhancement in SFRS sensitivity and precision can be achieved when the photon energy of the exciting laser radiation $\left(\hbar \omega_{L}\right)$ passes through the exci- 




FIG. 1. The Stokes (S) and anti-Stokes (AS) electron spin-flip Raman transitions of CdTe recorded at $6 \mathrm{~K}$ and $60 \mathrm{kG}$. (kcps $\equiv$ kilocounts/s.)

tonic band gap of the semiconductor and the SFRS efficiency is resonantly increased by orders of magnitude.

It is known that there are two contributions to the Zeeman splitting of the conduction and valence bands in DMS's [see, for instance, Eq. (3.3) in Ref. 8]: "intrinsic," i.e., band structure related, and that due to the exchange interactions. At high TMI concentrations in wide band gap DMS's, the exchange term totally dominates the Zeeman splitting, whereas at low TMI concentrations, the two contributions become comparable and one has to account for the intrinsic part in order to extract the $s p-d$ exchange interaction. At low TMI concentrations one can closely approximate the intrinsic properties of a DMS with those of the host material (e.g., $\mathrm{CdTe}$ is the host material for diluted magnetic $\mathrm{Cd}_{1-x} \mathrm{Mn}_{x} \mathrm{Te}$ ). The difference in the SFRS in a DMS and in the host should yield the $s p-d$ contribution with high precision.

In the present paper we report the SFRS results on CdTe and $\mathrm{Cd}_{1-x} \mathrm{~V}_{x} \mathrm{Te}$ and their interpretation and analysis aided by the characterization of the specimens with modulated reflectivity and magnetization. The modulated reflectivity provided important information about alloy formation and bandgap energy, while magnetization measurements enabled us to extract the exchange constant from SFRS.

\section{EXPERIMENT}

Bulk crystals of CdTe and $\mathrm{Cd}_{1-x} \mathrm{~V}_{x} \mathrm{Te}$ studied in the present investigation were grown by the modified vertical Bridgman method. Samples were cleaved from the boules perpendicular to [110]. Magnetization measurements on $\mathrm{Cd}_{1-x} \mathrm{~V}_{x} \mathrm{Te}$ samples were performed, employing a Quantum design MPMS XL7 superconducting quantum interference device, in the temperature range from $2-300 \mathrm{~K}$ in a magnetic field with the strength up to $70 \mathrm{kOe}$, the magnetic field being perpendicular to the cleaved surfaces. Using wavelength modulation, reflectivity spectra were obtained at low temperatures without an external magnetic field.

Raman spectra were excited with radiation from a tunable Ti:Sapphire laser pumped with a $\mathrm{Ar}^{+}$laser. The laser photon energy $\hbar \omega_{L}$ was tuned into resonance with the excitonic transitions of the samples. Special care was taken to prevent local heating and the photoionization of $\mathrm{V}$ ions in $\mathrm{Cd}_{1-x} \mathrm{~V}_{x} \mathrm{Te}$ by reducing the exciting radiation intensity until the spectral position of the SFRS transition was no longer affected; typical intensity did not exceed $2 \mathrm{~W} / \mathrm{cm}^{2}$. The scattered radiation was analyzed with a Spex double or, when a greater stray light rejection is desired, a triple spectrometer and detected using standard photon-counting electronics.

SFRS spectra were recorded in a $90^{\circ}$ scattering geometry with circularly polarized laser light incident along the direction of magnetic field $\hat{\mathbf{z}}$ and the scattered radiation analyzed with a linear polarizer along the magnetic field. The two scattering polarization configurations, $\left(\hat{\sigma}_{+}, \hat{\mathbf{z}}\right)$ and $\left(\hat{\sigma}_{-}, \hat{\mathbf{z}}\right)$, allow the SFRS polarization selection rules to be verified.

\section{RESULTS AND DISCUSSION}

\section{A. CdTe}

Figure 1 shows a part of the Raman spectrum of CdTe recorded at $6 \mathrm{~K}$ and $60 \mathrm{kG}$ near the photon energy of the exciting laser line. A pair of "red" shifted (Stokes, S) and "blue" shifted (anti-Stokes, AS) Raman lines is observed, with the Raman shift independent of temperature over the range $1.8-10 \mathrm{~K}$ and linear in magnetic field. The Raman lines are attributed to the electron spin-flip Raman transition. The SFRS shift in CdTe is given by

$$
\hbar \omega_{S F R S}=g^{*} \mu_{B} B,
$$

where $g^{*}$ is the appropriate $g$ factor, $\mu_{B}$ is the Bohr magneton, and $B$ is the magnetic induction. The least-squares linear fit to the dependence of the SFRS shift on $B$ yields $|g *|$ $=1.676 \pm 0.007$.

The sign of the $g$ factor can be determined from the polarization features observed in a right-angle scattering geometry schematically outlined in the inset of Fig. 2 . With the magnetic field along $\hat{\mathbf{z}}, \quad \hat{\sigma}_{+}=(1 / \sqrt{2})(\hat{\mathbf{x}}+i \hat{\mathbf{y}}) \quad$ and $\quad \hat{\sigma}_{-}$ $=(1 / \sqrt{2})(\hat{\mathbf{x}}-i \hat{\mathbf{y}})$, the circularly polarized incident radiation of positive and negative helicities, respectively, and the scattered radiation analyzed along $\hat{\mathbf{z}}$ yield the $\left(\hat{\sigma}_{+}, \hat{\mathbf{z}}\right)$ and $\left(\hat{\sigma}_{-}, \hat{\mathbf{z}}\right)$ polarization configurations. A Stokes component much stronger than the anti-Stokes is seen in $\left(\hat{\sigma}_{-}, \hat{\mathbf{z}}\right)$ (Fig. 2), whereas in $\left(\hat{\sigma}_{+}, \hat{\mathbf{z}}\right)$ it is opposite. The appearance of the weak antiStokes component in $\left(\hat{\sigma}_{-}, \hat{\mathbf{z}}\right)$ and that of the weak Stokes component in $\left(\hat{\sigma}_{+}, \hat{\mathbf{z}}\right)$ are consequences of the small scattering volume probed in the near resonance condition, resulting in unavoidable departures from the exact $\left(\hat{\sigma}_{ \pm}, \hat{\mathbf{z}}\right)$ configurations. Within this limitation, the distinctly stronger Stokes in $\left(\hat{\sigma}_{-}, \hat{\mathbf{z}}\right)$ and anti-Stokes in $\left(\hat{\sigma}_{+}, \hat{\mathbf{z}}\right)$ are strong evidence for ascribing a negative sign to the $g$ factor.

We note that $g^{*}=-1.676 \pm 0.007$ is in excellent agreement with $1.6800 \leqslant|g *| \leqslant 1.6808$ for the conduction electrons in CdTe obtained from a microwave electron paramagnetic resonance (EPR) experiment ${ }^{9}$ and close to the 


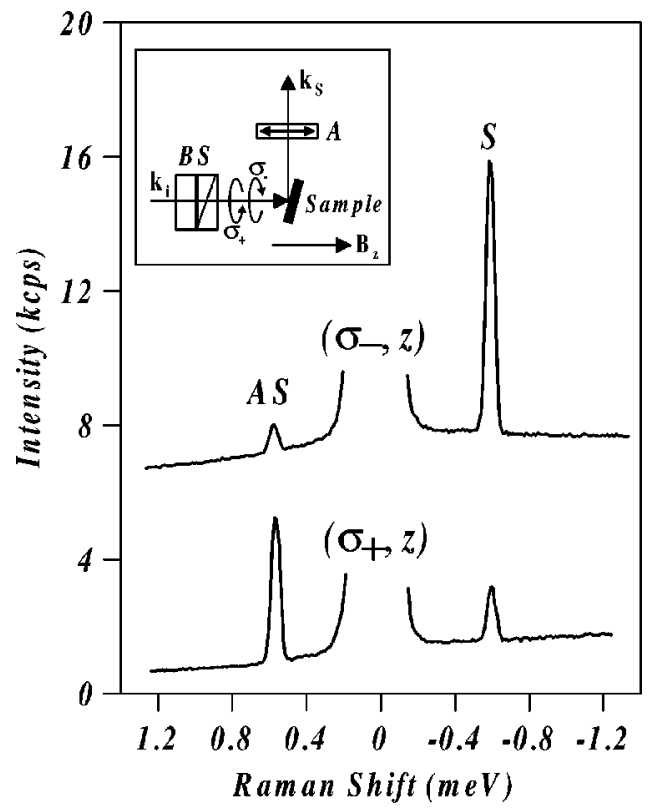

FIG. 2. Electron SFRS spectra of CdTe recorded in $\left(\hat{\sigma}_{+}, \hat{\mathbf{z}}\right)$ and $\left(\hat{\sigma}_{-}, \hat{\mathbf{z}}\right)$ polarization configurations at $6 \mathrm{~K}$ and $60 \mathrm{kG}$. The inset shows the scattering geometry; BS is a Babinet-Soleil compensator, A, a linear analyzer.

theoretical value of -1.64 calculated by Willatzen et al. ${ }^{10} \mathrm{It}$ is equally noteworthy that Nakamura et al. ${ }^{11}$ reported a $g$ factor of $-1.59 \pm 0.02$ with optically detected electron spin resonance, Oestreich et al. ${ }^{12}$ obtained $g^{*}=-1.653 \pm 0.02$ in a spin quantum beats experiment, while Simmonds et al. ${ }^{13}$ measured $g^{*}=-1.6$ from the magnetoluminescence of an exciton bound to a shallow donor. In contrast, an early electron SFRS experiment ${ }^{14}$ yielded a $g$ factor $-0.74 \pm 0.03$, clearly inconsistent with the values obtained with the variety of experimental techniques and the theoretical calculation.

A deviation of the electron $g$ factors in semiconductors from +2 , the electron $g$ factor in vacuum, is a well known "band structure" effect arising from the mixing of electronic bands by the spin-orbit interaction. The effect is especially striking in narrow-gap semiconductors, e.g., $\mathrm{InSb}^{15}$ and $\mathrm{Hg}_{1-x} \mathrm{Mn}_{x} \mathrm{Te},{ }^{16}$ in which the electron $g$ factors reach values as low as -50 . Although departure of the electron $g$ factor in CdTe from +2 is not as dramatic as those in the narrow-gap semiconductors, it unambiguously shows the importance of the band structure effects even in wide-gap semiconductors.

In order to identify and delineate the electronic transitions and the Raman mechanisms responsible for the electron SFRS, we investigated the intensity of the Raman line as a function of $\hbar \omega_{L}$ and that of the scattered photon energy $\left(\hbar \omega_{S}\right)$. The resulting resonance profiles measured at $6 \mathrm{~K}$ and $60 \mathrm{kG}$ are displayed in Fig. 3. Two distinct resonances for the Stokes, as well as the anti-Stokes component, are clearly visible. The stronger resonance peaks of both components occur in the spectral range in which magnetoluminescence lines due to recombination of an exciton bound to a shallow neutral donor have been reported. ${ }^{17} \mathrm{We}$, therefore, attribute them to a spin-flip Raman process mediated via an exciton bound to a shallow neutral donor. ${ }^{18}$ Although the exact

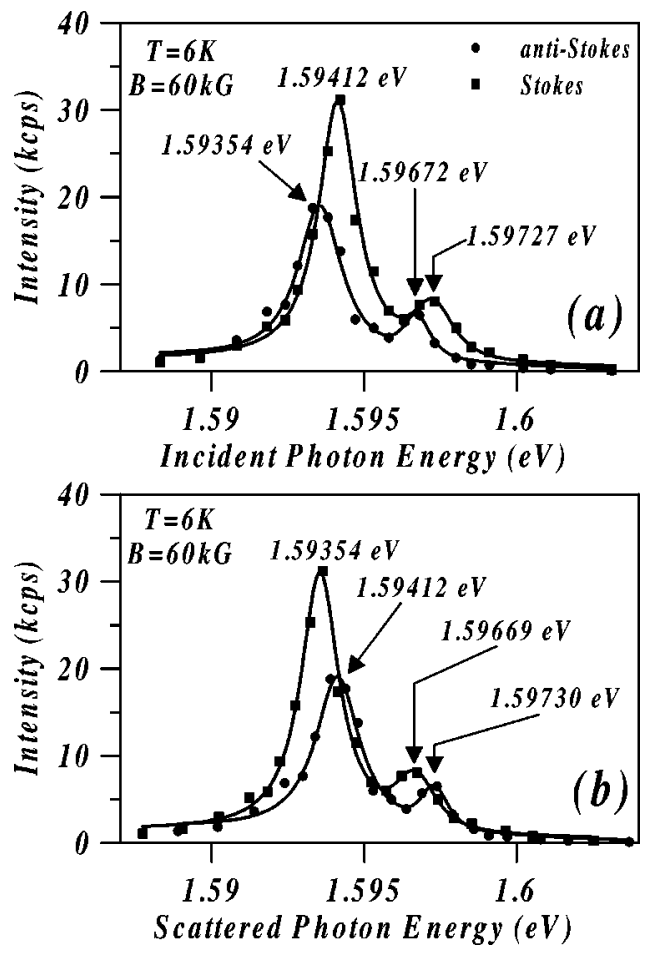

FIG. 3. Resonance profiles of Stokes and Anti-Stokes components of electron SFRS in CdTe as functions of (a) incident and (b) scattered photon energies. Circles and squares are experimental data. Solid curves are fits to the data using Eqs. (2) and (3), yielding energy positions of the resonance peaks.

chemical nature of the shallow donor has yet to be established, the binding energy of the donor-bound exciton is consistent with those measured for effective mass donors in CdTe. ${ }^{19}$

On the basis of their spectral positions, which coincide within the experimental uncertainties with the Zeeman transitions of free exciton observed in reflectivity, ${ }^{20}$ the weaker resonance maxima in Fig. 3 can be attributed to a spin-flip Raman process mediated via free excitonic levels. We note that the zero-field free exciton energy measured in the present study using the wavelength modulated reflectivity (see Fig. 4) is $1.5966 \mathrm{eV}$ consistent with the value reported by Neumann et al. ${ }^{21}$ whereas Dreybrodt et al. ${ }^{20}$ estimated their zero-field exciton energy to be $1.5949 \mathrm{eV}$. Thus, magnetoreflectivity data of Dreybrodt et al. have to be shifted by $1.7 \mathrm{meV}$ to higher energies to be consistent with the present Raman resonance profiles.

The resonances are rather broad with a full width at the half maximum of about $2 \mathrm{meV}$. Very close to the resonance maxima the polarization selection rules are not strictly obeyed, as noted by Oka and Cardona ${ }^{22}$ in ZnTe and by Scott et $a .^{23}$ in CdS.

The Raman process mediated via an exciton bound to a neutral donor is depicted schematically in Fig. 5(a). The initial and final states are the Zeeman components of the ground state of the neutral donor $(D)$. The intermediate state is one of the Zeeman levels derived from the ground state of an exciton bound $(B X)$ to the neutral donor. Notice that the Zeeman splitting of $B X$ is that of its hole, since its two 


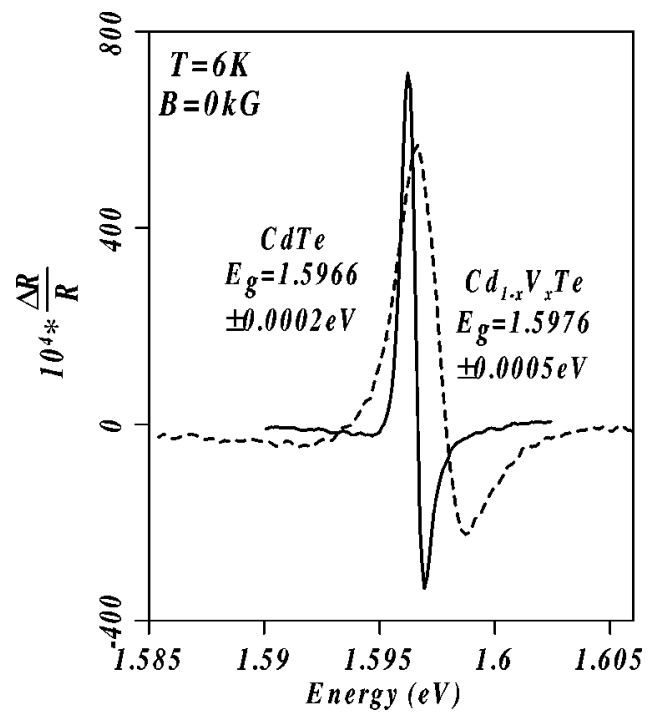

FIG. 4. Wavelength modulated reflectivity spectra of CdTe (solid line) and $\mathrm{Cd}_{1-x} \mathrm{~V}_{x} \mathrm{Te}$ (dashed line) at zero magnetic field.

electrons have antiparallel spins in its lowest-energy state; the ordering of the Zeeman levels is based on the positive sign of the $g$ factor of the hole. ${ }^{21,24}$ The Stokes transition in this process occurs as follows: initially, the neutral donor is in its $\left|\frac{1}{2}\right\rangle_{D}$ state; the incident $\hat{\sigma}_{-}$radiation couples $\left|\frac{1}{2}\right\rangle_{D}$ with the $\left|-\frac{1}{2}\right\rangle_{B X}$ excitonic state, while the scattered radiation with polarization $\hat{\mathbf{z}}$ couples $\left|-\frac{1}{2}\right\rangle_{B X}$ with $\left|-\frac{1}{2}\right\rangle_{D}$, resulting in a Stokes SFRS transition from $\left|\frac{1}{2}\right\rangle_{D}$ to $\left|-\frac{1}{2}\right\rangle_{D}$. The antiStokes transition can be similarly described.

The differential scattering cross section for such two-step process can be written in the following form (see, for instance, Ref. 25):

$$
\frac{d \sigma}{d \Omega} \approx f^{2}\left(\frac{e^{2}}{m c^{2}}\right)^{2} \frac{\omega_{s}}{\omega_{i}} \frac{\left(\hbar \omega_{i}\right)^{2}}{\left(E-\hbar \omega_{i}\right)^{2}+(\Gamma / 2)^{2}} .
$$

Here, $f$ is the oscillator strength of the transition $D \rightarrow B X$; $\hbar \omega_{i}$, the incident photon energy; $E$, an energy of the transition $D \rightarrow B X$ (e.g., $E\left(\left|-\frac{1}{2}\right\rangle_{B X}\right)-E\left(|1 / 2\rangle_{D}\right)$ for the Stokes transition); and $\Gamma$, a phenomenological damping term.

Equation (2) implies a single "in-resonance" for the twostep process when $\hbar \omega_{i}$ equals $E$. On the basis of the Zeeman splittings in Fig. 5(a) one expects that the Stokes resonance should occur at an incident photon energy higher than that for the anti-Stokes, with a separation given by $\Delta-\delta$, where $\Delta$ and $\delta$ are separations of the Zeeman levels of the neutral donor and the neutral donor-bound exciton, respectively. While the occurrence of the Stokes resonance peak at a higher energy $(1.59412 \mathrm{eV})$ compared to that of anti-Stokes
Donor-Bound Exciton

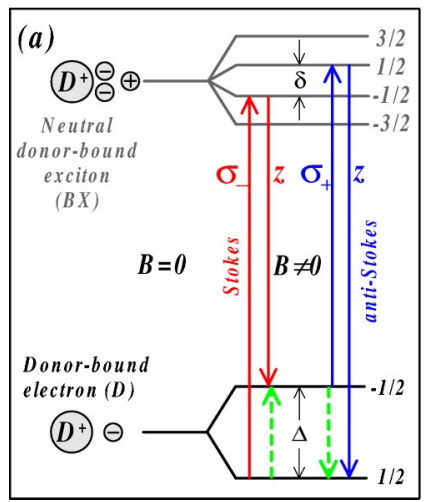

Free Exciton

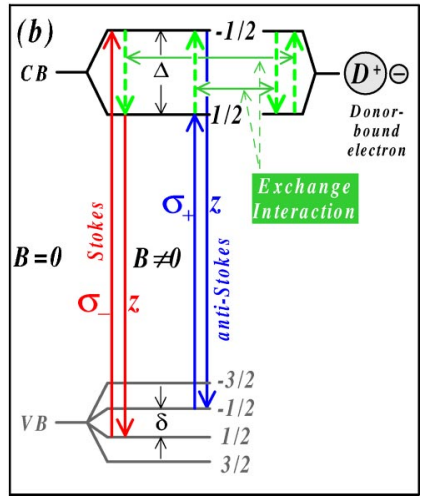

FIG. 5. (Color online). Electron SFRS processes mediated via (a) an exciton bound to a neutral donor and (b) a free exciton.

resonance peak at a lower energy $(1.59354 \mathrm{eV})$ follows from Fig. 3(a), the experimentally observed separation of the resonances is equal to $\Delta$. We note that at $60 \mathrm{kG}, \delta$ should be $\sim 0.2 \mathrm{meV}^{21,24}$ With the typical width of the donor-bound exciton level being $\sim 0.1 \mathrm{meV},{ }^{19}$ it appears that the Zeeman components of the bound exciton in Fig. 5(a) merge into a single broad band, consistent with the observed separation of the stronger SFRS resonances in Fig. 3(a).

In analogy to the Raman-EPR transition of $\mathrm{Mn}^{2+}$ in $\mathrm{Cd}_{1-x} \mathrm{Mn}_{x} \mathrm{Te}^{26}$ one can ascribe the weaker resonances to a three-step Raman process mediated via interband free excitonic transitions. Figure 5(b) shows the $\frac{3}{2}, \frac{1}{2},-\frac{1}{2},-\frac{3}{2}$ magnetic sublevels of the $\Gamma_{8}$ valence band $(V)$ (the ordering being that of the valence band electron) and the $-\frac{1}{2}, \frac{1}{2}$ magnetic sublevels of the $\Gamma_{6}$ conduction band $(C)$. The excitonic Zeeman levels are constructed from these valence and conduction band sublevels with the Coulomb interaction between the electron and hole being taken into account appropriately. In the $\left(\hat{\sigma}_{-}, \hat{\mathbf{z}}\right)$ Stokes scattering configuration, the three steps leading to the electron spin flip are: (i) the incident $\hat{\sigma}_{-}$radiation couples the $\left|\frac{1}{2}\right\rangle_{V}$ state with the $\left|-\frac{1}{2}\right\rangle_{C}$ state, (ii) an exchange interaction between the conduction electron and a donor electron induces a mutual spin flip in them; and (iii) finally, the $\hat{\mathbf{z}}$-polarized scattered radiation couples the $\left|\frac{1}{2}\right\rangle_{C}$ state with the original $\left|\frac{1}{2}\right\rangle_{V}$ state. In this manner, the Stokes shift is associated with the spin flip of the donor electron from its $\left|\frac{1}{2}\right\rangle_{D}$ initial to $\left|-\frac{1}{2}\right\rangle_{D}$ final state. The anti-Stokes spin-flip transition of the donor electron $\left|-\frac{1}{2}\right\rangle_{D}$ $\rightarrow\left|\frac{1}{2}\right\rangle_{D}$ achieved with $\left(\hat{\sigma}_{+}, \hat{\mathbf{z}}\right.$ polarization can be similarly described, but with the incident (scattered) transition originating (terminating) at the $\left|-\frac{1}{2}\right\rangle_{V}$ state.

Adapting Loudon's theory for optical phonons, ${ }^{27}$ the differential scattering cross section for Stokes component of such a three-step process can be written as

$$
\frac{d \sigma}{d \Omega} \propto\left|\frac{\left\langle\frac{1}{2}^{V}, n_{S}+1\left|\hat{H}_{e R}\right| \frac{1}{2}^{C}, n_{S}\right\rangle\left\langle-\frac{1}{2}^{D}, \frac{1}{2}^{C}\left|\hat{H}_{e x}\right| \frac{1}{2}^{D},-\frac{1}{2}^{C}\right\rangle\left\langle-\frac{1}{2}^{C}, n_{I}-1\left|\hat{H}_{e R}\right| \frac{1}{2}^{V}, n_{I}\right\rangle}{\left[\hbar \omega_{I}-E\left(\left|\frac{1}{2}{ }^{V},-\frac{1}{2}^{C}\right\rangle_{X}\right)\right]\left[\hbar \omega_{I}-\hbar \omega_{S F R S}-E\left(\left|\frac{1}{2}^{V}, \frac{1}{2}^{C}\right\rangle_{X}\right)\right]}+\cdots\right|^{2},
$$


where the ellipsis represents five additional terms. In this expression, $\hat{H}_{e x}$ is the exchange interaction Hamiltonian between the electrons, superscripts $V, C$, and $D$ stand for valence, conduction and donor states, respectively, $n_{I}$ and $n_{S}$, numbers of incident and scattered photons, respectively, before the scattering process, and $E_{X}$, an energy of an excitonic Zeeman sublevel.

The two terms in the denominator of Eq. (3) should result in an in-resonance and an out-resonance in the scattering cross section. However, only one such resonance is observed at the free exciton energy for the Stokes, as well as for the anti-Stokes, a consequence of the coincidence of the in- and out-resonance conditions; such a situation has been referred to as a double resonance in the literature. ${ }^{28,29}$ In order for this double-resonance condition to be maintained independent of $\mathbf{H}$, the Zeeman splitting of the conduction band and that of the donor electron must equal. For a shallow donor with effective mass wave function such an equality is to be expected.

The Zeeman splitting scheme of the free exciton shown in Fig. 5(b) implies that in-resonance of the Stokes transition and that of the anti-Stokes should be separated by $\Delta+\delta$, whereas the separation of the corresponding out-resonances will be $\Delta-\delta$. While the relative spectral positions of the weaker resonance maxima in Fig. 3 agree with the splitting scheme, the separations of the in, as well as out, resonances equal $\Delta$. As in the case of the bound-exciton resonances considered above, this can be explained by a small $\delta$, which results in an overlap of the valence band Zeeman levels, in turn producing the observed separations of the weaker SFRS resonances in Fig. 3.

The photoluminescence spectra from the CdTe samples investigated displayed a strong peak at $1.5896 \mathrm{eV}$ associated with a recombination of an exciton bound to an anonymous shallow neutral acceptor, while the series of peaks in the range from 1.5930 to $1.5959 \mathrm{eV}$, usually attributed to the recombination of an exciton bound to shallow neutral donors, is absent. ${ }^{19}$ Hence, the electron SFRS observed in the samples studied could very well originate from a steady-state photoexcited population of neutral donors and/or conduction electrons, as in the case of the electron SFRS reported in p-type $\mathrm{Cd}_{1-x} \mathrm{Mn}_{x} \mathrm{Te}^{30}$

\section{B. $\mathrm{Cd}_{1-x} \mathrm{~V}_{x} \mathrm{Te}$}

In this section we report and discuss spin-flip Raman scattering from shallow donors in $\mathrm{Cd}_{1-x} \mathrm{~V}_{x} \mathrm{Te}$ and its magnetic field/temperature dependence analyzed in terms of magnetization. In Fig. 4, the wavelength modulated reflectivity spectrum of CdTe is compared to that of a $\mathrm{Cd}_{1-x} \mathrm{~V}_{x}$ Te specimen; the $1 \pm 0.7-\mathrm{meV}$ shift of the excitonic band gap of the latter demonstrates that it is indeed a ternary alloy.

Substitutional vanadium on a $\mathrm{Cd}$ site in $\mathrm{CdTe}$ is an isoelectronic impurity. When the two $4 s$ electrons of [Ar] $3 d^{3} 4 s^{2}$ electronic configuration complete the tetrahedral bonds with the neighboring Te ions, the charge state of $\mathrm{V}$ is $\mathrm{V}^{2+}$. It has been reported that substitutional $\mathrm{V}$ introduces deep donor and acceptor electronic states in II-VI DMS's, ${ }^{31}$ the former arising from an electron contributed by the $3 d$ shell and the latter due to an electron accepted in it. The position of the $\mathrm{V}^{2+} / \mathrm{V}^{3+}$-donor ${ }^{32}$ level has been estimated $^{33-35}$ to be in the range from $\mathrm{E}_{C B}-0.78 \mathrm{eV}$ to $\mathrm{E}_{C B}-0.67 \mathrm{eV}$, while the $\mathrm{V}^{2+} / \mathrm{V}^{+}$-acceptor level has been determined $^{36}$ to be at $\mathrm{E}_{V B}+1.0 \mathrm{eV}\left(E_{C B}\right.$ and $E_{V B}$ are conduction band minimum and valence-band maximum energies, respectively).

In $\mathrm{CdTe}$, depending on the specific growth conditions, native donors or native acceptors are known to occur; ${ }^{37}$ in addition, inadvertently introduced chemical impurities can also behave as residual shallow donors (acceptors). ${ }^{37}$ Under these circumstances, $\mathrm{V}^{2+}$ can be compensated to yield either $\mathrm{a}^{+}$or a $\mathrm{V}^{3+}$ charge state. Indeed, the microwave EPR experiments on CdTe: $\mathrm{V}$ revealed ${ }^{38,39}$ the signature of $\mathrm{V}^{3+}$ centers, which could be converted into that characteristic of $\mathrm{V}^{2+}$ with photon energies higher than $0.93 \mathrm{eV}^{35}$ whereas Schwartz et al. ${ }^{39}$ and Slodowy and Baranowski ${ }^{40}$ measured absorption spectra of $\mathrm{CdTe}: \mathrm{V}$ which they attributed to the internal transitions of $\mathrm{V}^{2+}$.

In order to interpret the magnetic field $(B)$ and temperature $(T)$ dependence of SFRS of an electron bound to a shallow donor in $\mathrm{Cd}_{1-x} \mathrm{~V}_{x} \mathrm{Te}$, the charge state of $\mathrm{V}$ and its corresponding ground state (inclusive of crystal field, spin-orbit coupling and possible Jahn-Teller effects) are significant. Each of the charge states is expected to display a distinctive magnetization with a characteristic dependence on $B$ and $T$. In the following we delineate the distinguishing features expected in the magnetic behavior of $\mathrm{V}^{3+}, \mathrm{V}^{2+}$, and $\mathrm{V}^{+}$. The experimental results will allow us to identify the charge state which accounts for them.

Villeret et al. ${ }^{41}$ have shown that the ${ }^{3} \mathrm{~F}$ ground state of $\mathrm{V}^{3+}$ free ion is split into three levels ${ }^{3} \Gamma_{2},{ }^{3} \Gamma_{5}$, and ${ }^{3} \Gamma_{4}$ by a tetrahedral crystal field (see Fig. 7 in Ref. 41) with the orbital singlet ${ }^{3} \Gamma_{2}$ as the ground state; to first order in spinorbit interaction, this level becomes the three-fold degenerate $\Gamma_{2} \otimes \Gamma_{4}=\Gamma_{5}$ level. Zeeman splitting of $\Gamma_{5}$ is isotropic with a $g$ factor given by $2+(32 \lambda / 5 \Delta)$, where $\lambda$ is a spin-orbit interaction constant and $\Delta$ the crystal-field splitting parameter. Thus, the low temperature magnetization associated with $\mathrm{V}^{3+}$ ions in the tetrahedral crystal field is expected to display an isotropic paramagnetic behavior described by the $\mathcal{B}_{1}$ Brillouin function. Since the present study of $\mathrm{Cd}_{1-x} \mathrm{~V}_{x} \mathrm{Te}$ is limited to small $x, \mathrm{~V}$ ions are assumed to be well separated; therefore, interactions between them can be neglected and their magnetization well described by Brillouin functions, rather than by modified Brillouin functions.

In contrast, the ground state of $\mathrm{V}^{2+}$ ion in a tetrahedral crystal field is an orbital triplet ${ }^{4} \Gamma_{4}$ (see Fig. 6 in Ref. 41). Such a state is known to be split by a Jahn-Teller distortion which lifts (partially or totally) the orbital degeneracy, resulting in the ground state being an orbital singlet ${ }^{4} \boldsymbol{\Gamma}_{2}$ with a four-fold spin degeneracy. ${ }^{35}$ In the absence of a magnetic field, the spin-orbit interaction further splits the ground state into two Kramers doublets with spin projections $\pm 1 / 2$ and $\pm 3 / 2$, respectively. Christmann et al. ${ }^{35}$ estimated the splitting to be in excess of $1 \mathrm{~cm}^{-1}$. If the separation between the Kramers doublets is sufficiently large, so that only the $\pm \frac{1}{2}$ doublet need be considered, then the magnetization will be- 


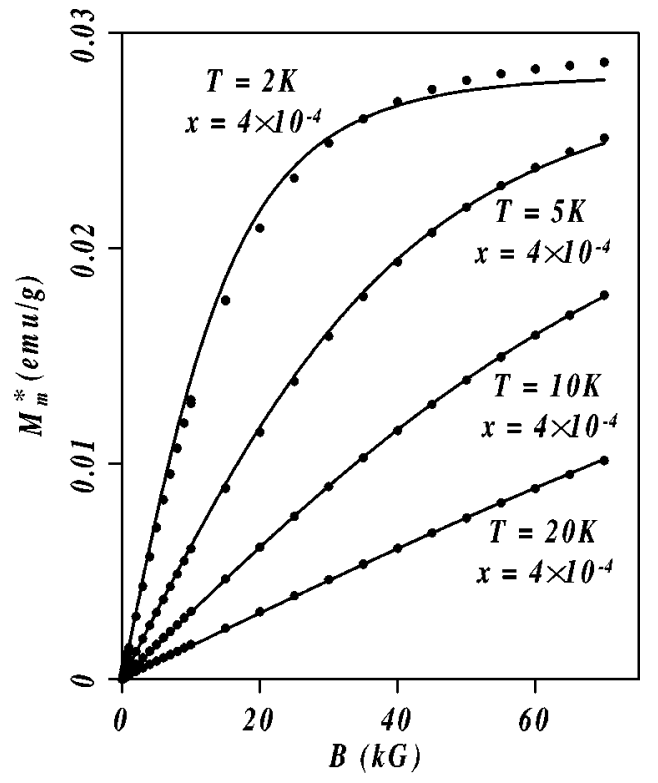

FIG. 6. Magnetization of $\mathrm{Cd}_{1-x} \mathrm{~V}_{x}$ Te corrected for host diamagnetism as a function of magnetic field at several temperatures. Solid circles are experimental data, and lines, the least squares $\mathcal{B}_{3 / 2}$ Brillouin function fits. The plot shows experimental temperatures and $\mathrm{V}^{2+}$ concentrations deduced from the fits. See text for details.

have as the $\mathcal{B}_{1 / 2}$ Brillouin function. However, if the separation is small, the magnetization will follow $\mathcal{B}_{3 / 2}$.

We note that $\mathrm{V}^{+}$ion has the same electronic configuration as $\mathrm{Cr}^{2+}$, therefore one expects them to have the same electronic energy structure in $\mathrm{CdTe}$, hence the same type of magnetization. A theoretical model, ${ }^{42}$ with crystal-field effects and Jahn-Teller distortion duly taken into account, predicts the ground state of the $\mathrm{Cr}^{2+}$ ion in the tetrahedral environment to be an orbital singlet with spin 2. This is further split by spin-orbit and spin-spin interactions. Magnetic measurements on $\mathrm{Cd}_{1-x} \mathrm{Cr}_{x} \mathrm{Te}$ have shown ${ }^{43}$ however, that the magnetization of $\mathrm{Cr}^{2+}$ is isotropic with a $B / T$ dependence characteristic of the $\mathcal{B}_{2}$ Brillouin function. This behavior indicates that the spin related splittings can be neglected.

Magnetization of the $\mathrm{Cd}_{1-x} \mathrm{~V}_{x} \mathrm{Te}$ sample, corrected for the host diamagnetism, i.e., associated with $\mathrm{V}$ ions only $\left(M_{m}^{*}\right)$, is displayed in Fig. 6 in solid circles as a function of magnetic field at four different temperatures, $B$ being along [110]. The low-field $(<10 \mathrm{kG})$ magnetic susceptibility $\chi$ $=\left(M_{m}^{*} / B\right)$ deduced from the data displays the characteristic $(C / T)$ Curie dependence (Fig. 7), with $C=(3.1686$ $\pm 0.0492) \times 10^{-6} \mathrm{~cm}^{3} \mathrm{~K} / \mathrm{g}$. Note $C$ the Curie constant, is given by

$$
C=\frac{\left(g_{i} \mu_{B}\right)^{2} x S(S+1) N_{A}}{3 k_{B} W}
$$

At high magnetic fields and low temperatures, one expects all magnetic moments of magnetic ions to be oriented along the external magnetic field, resulting in the saturation of the magnetization at the theoretical value given by

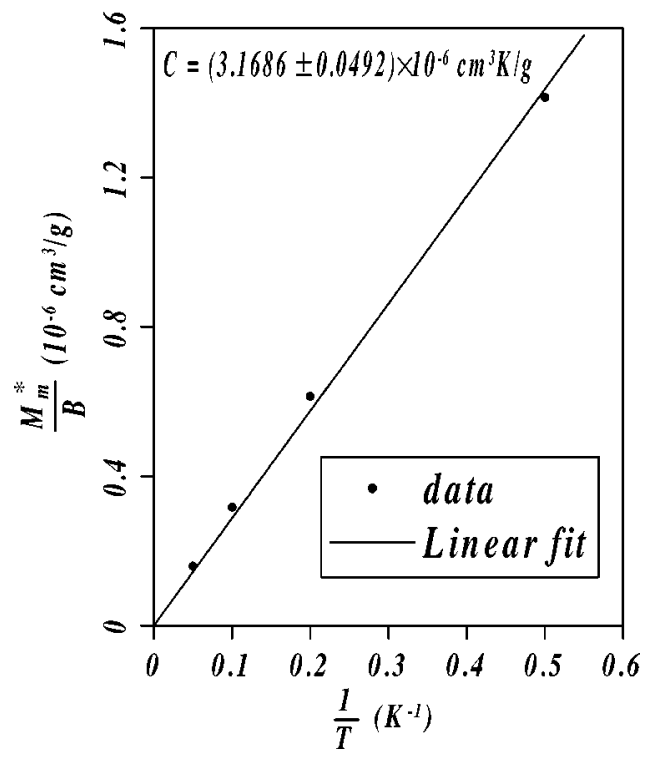

FIG. 7. $\left(M_{m}^{*} / B\right)$ vs $(1 / T)$ at low magnetic fields, where $M_{m}^{*}$ is the magnetization due to $\mathrm{V}$ ions.

$$
M_{S A T}^{*}=\frac{g_{i} \mu_{B} x S N_{A}}{W}
$$

It follows from Eqs. (4) and (5):

$$
\frac{C}{M_{S A T}^{*}}=\frac{g_{i} \mu_{B}(S+1)}{3 k_{B}} .
$$

The value for $M_{S A T}^{*}$ estimated from Fig. 6 is 0.02887 $\pm 0.00047 \mathrm{emu} / \mathrm{g}$, yielding $(S+1)=2.45 \pm 0.08$ from Eq. (6). This is in excellent agreement with the spin of the $\mathrm{V}^{2+}$ charge state.

Furthermore, the magnetization data were fitted with the $\mathcal{B}_{3 / 2}$ Brillouin function (solid curves in Fig. 6) according to

$$
M_{m}^{*}=\frac{g_{i} \mu_{B} N_{A} x S_{i}}{W\left(\mathrm{Cd}_{1-x} \mathrm{~V}_{x} \mathrm{Te}\right)} \mathcal{B}_{3 / 2}\left(\frac{S_{i} g_{i} \mu_{B} B}{k_{B} T}\right),
$$

where $g_{i}=g\left(\mathrm{~V}^{2+}\right) \approx 2$, the $g$ factor of $\mathrm{V}^{2+}$ ion in CdTe; $S_{i}$ $=S\left(\mathrm{~V}^{2+}\right)=3 / 2$, the spin of $\mathrm{V}^{2+}$ ion; $W\left(\mathrm{Cd}_{1-x} \mathrm{~V}_{x} \mathrm{Te}\right)$ $\approx W(\mathrm{CdTe})=240 \mathrm{~g} / \mathrm{mole}$, the molar weight of the $\mathrm{Cd}_{1-x} \mathrm{~V}_{x} \mathrm{Te}$ sample; here $x$, the $\mathrm{V}^{2+}$ molar concentration, is the only fitting parameter. These fits yielded the same value for $x(\approx 0.0004)$ for all the four temperatures. In contrast, fits with $\mathcal{B}_{1 / 2}, \mathcal{B}_{1}$, and $\mathcal{B}_{2}$ Brillouin functions resulted in significantly differing values of $x$ at different temperatures, with discrepancies as large as $10 \%, 20 \%$, and $40 \%$, respectively, and were qualitatively unsatisfactory. On this basis we conclude that the significant majority of the vanadium ions in the $\mathrm{Cd}_{1-x} \mathrm{~V}_{x} \mathrm{Te}$ investigated are in the $\mathrm{V}^{2+}$ charge state. The $\mathrm{V}^{2+}$ concentration determined from the fits is 5.9 $\times 10^{18} \mathrm{~cm}^{-3}$, several orders higher than typical concentrations of the native defects and shallow chemical impurities incorporated in the crystal during the growth. ${ }^{37}$ Under these 


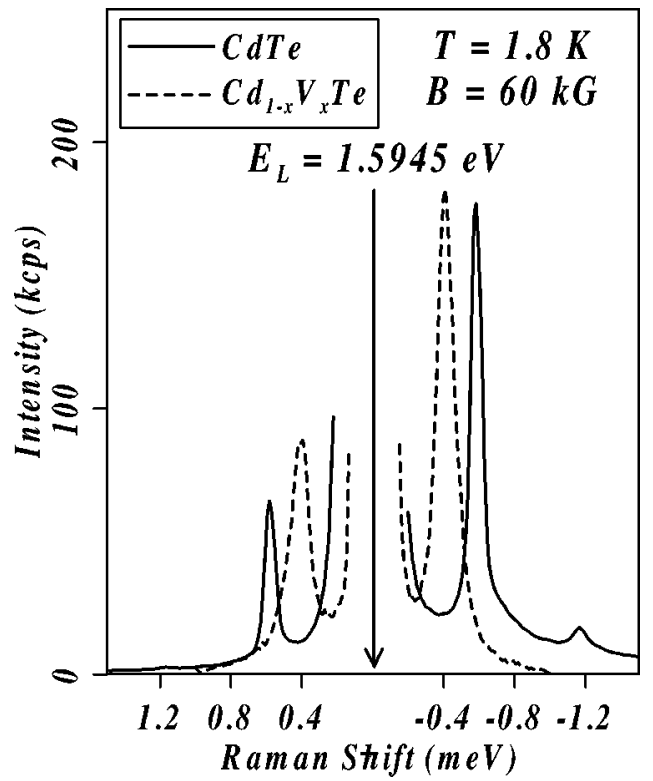

FIG. 8. Electron SFRS spectra of CdTe and $\mathrm{Cd}_{1-x} \mathrm{~V}_{x} \mathrm{Te}$.

circumstances, one indeed expects most of the $\mathrm{V}$ ions to be in the $\mathrm{V}^{2+}$ charge state, in agreement with the magnetization measurements.

In Fig. 8 electron SFRS transitions in CdTe and $\mathrm{Cd}_{1-x} \mathrm{~V}_{x} \mathrm{Te}$, recorded at $1.8 \mathrm{~K}$ and $60 \mathrm{kG}$, are compared. The apparent decrease in the Raman shift in the latter is due to a strong $s$ - $d$ exchange interaction between the $s$-like conduction electrons and the $3 d$ shell of $\mathrm{V}^{2+}$ ions, present in $\mathrm{Cd}_{1-x} \mathrm{~V}_{x} \mathrm{Te}$, but not in CdTe.

Following Kossut, ${ }^{44}$ the exchange interaction Hamiltonian can be written in the Heisenberg form, i.e.,

$$
\hat{H}_{s d}=-\sum_{i} J^{s d}\left(\mathbf{r}-\mathbf{R}_{i}\right) \mathbf{S}_{i} \cdot \mathbf{s}
$$

where $J^{s d}$ is the $s$ - $d$ exchange integral, $\mathbf{r}$ and $\mathbf{s}$ are the electron position and spin operators, $\mathbf{R}_{i}$ and $\mathbf{S}_{i}$ are the $3 d$-transition metal ion position and spin operators, and the summation is over all lattice sites occupied by the TMI's.

A treatment of the band structure problem with the Hamiltonian given by Eq. (8) represents a theoretical challenge, because the Hamiltonian does not posses the translational symmetry of a perfect crystal. However, two approximations considerably simplify the situation. (i) Due to the extended nature of its wave function, the electron interacts with a large number of TMI's. Hence the molecular field approximation can be invoked to replace the TMI spin operator with its thermal and spatial average $\langle\langle\mathbf{S}\rangle\rangle$ taken over all the ions. (ii) The summation of the exchange coupling $J^{s d}\left(\mathbf{r}-\mathbf{R}_{i}\right)$ over the TMI sites $\mathbf{R}_{i}$ is replaced by a summation over all cation sites multiplied by the TMI mole fraction $x$. Considering a magnetically isotropic crystal in a magnetic field along, say, the $z$ axis, one gets $\left\langle\left\langle S_{x}\right\rangle\right\rangle=\left\langle\left\langle S_{y}\right\rangle\right\rangle=0$ and $\langle\langle\mathbf{S}\rangle\rangle=\left\langle\left\langle S_{z}\right\rangle\right\rangle$. Therefore, Eq. (8) transforms into

$$
\hat{H}_{s d}=s_{z} x\left\langle\left\langle S_{z}\right\rangle\right\rangle \sum_{\mathbf{R}} J^{s d}(\mathbf{r}-\mathbf{R}) .
$$



FIG. 9. $s-d$ exchange energy, deduced from electron SFRS for three different crystallographic directions of the magnetic field as a function of $(B / T)$.

The $s-d$ interaction described by Eq. (9) produces an electronic Zeeman splitting:

$$
\Delta_{C B}^{s d}=-x \alpha N_{0}\left\langle\left\langle S_{z}\right\rangle\right\rangle,
$$

over and above that due to the intrinsic electron $g$ factor given in Eq. (1). In Eq. (10), $N_{0}$ is the number of unit cells per unit volume and $\alpha=\left\langle s\left|J^{s d}\right| s\right\rangle$ is the $s$ - $d$ exchange integral for the conduction electron. Hence, the net Zeeman splitting of the conduction band minimum in $\mathrm{Cd}_{1-x} \mathrm{~V}_{x} \mathrm{Te}$ is given by

$$
\Delta_{C B}=g^{*} \mu_{B} B-x \alpha N_{0}\left\langle\left\langle S_{z}\right\rangle\right\rangle .
$$

Expressing the average spin $\left\langle\left\langle S_{z}\right\rangle\right\rangle$ in terms of the magnetization $M^{*}$ (Ref. 45):

$$
M_{m}^{*}=-\frac{g_{i} \mu_{B} N_{A}}{W\left(C d_{1-x} V_{x} T e\right)} x\left\langle\left\langle S_{z}\right\rangle\right\rangle,
$$

Eq. (11) transforms into

$$
\Delta_{C B}=g^{*} \mu_{B} B+\alpha N_{0} \frac{W\left(C d_{1-x} V_{x} T e\right)}{g_{i} \mu_{B} N_{A}} M_{m}^{*} \text {. }
$$

The second term in Eq. (13), referred to as $s$ - $d$ exchange energy, can be determined experimentally by measuring $\Delta_{C B}$ as a SFRS shift and assuming $g *$ in $\mathrm{Cd}_{1-x} \mathrm{~V}_{x}$ Te to be the same as that in CdTe. The negative $g^{*}$ together with the smaller Raman shift for the $\mathrm{V}^{2+}$ concentration in the specimen studied show that the $s-d$ interaction in $\mathrm{Cd}_{1-x} \mathrm{~V}_{x} \mathrm{Te}$ is ferromagnetic, i.e., $\alpha$ is positive.

The $s$ - $d$ exchange energy determined from the electron SFRS shifts is displayed as a function of $B / T$ in Fig. 9 with $B$ at $0^{\circ}, 45^{\circ}$, or $90^{\circ}$ with respect to (110). Scatter in the data could be due to a marginal anisotropy in the $s$ - $d$ exchange energy and/or the inhomogeneous distribution of vanadium ions; although further controlled experiments are needed to differentiate the two, we note that the anisotropy in magnetization, and hence that of the $s-d$ exchange interaction, for $\mathrm{V}^{2+}$ is not expected. ${ }^{35}$ The data exhibit $B / T$ dependence consistent with the $\mathcal{B}_{3 / 2}$ Brillouin function behavior charac- 




FIG. 10. The $s-d$ exchange energy vs magnetization. Dots are experimental data and the solid line is the least squares linear fit to them.

teristic of $M_{m}^{*}$ in $\mathrm{Cd}_{1-x} \mathrm{~V}_{x}$ Te. Figure 10 shows a plot of the $s-d$ exchange energy vs $M_{m}^{*}$. A linear least squares fit to the data according to Eq. (13) yields $\alpha N_{0}=(285 \pm 8) \mathrm{meV}$ in CdTe: $\mathrm{V}^{2+}$ assuming $g\left(\mathrm{~V}^{2+}\right)=2$ and $W\left(\mathrm{Cd}_{1-x} \mathrm{~V}_{x} \mathrm{Te}\right)$ $\approx W(\mathrm{CdTe})=240 \mathrm{~g} / \mathrm{mole}$.

\section{CONCLUSIONS}

The rich variety in the magnetic behavior of the $3 d$-TMI's, incorporated substitutionally on cation sites in the II-VI semiconductors, originates in (i) the number of electrons in the $3 d$ shell, (ii) effects of crystal field and spin-orbit interaction, and (iii) possible Jahn-Teller distortions. While theoretical studies have addressed all of these, experimental investigations have to contend with the severe limitation in the solubility of the TMI's in the II-VI DMS's, with the exception of $\mathrm{Mn}^{2+}$. It is in this context that the competing "intrinsic," band structure related, spin splittings of bands and that produced by the $s p$ - $d$ exchange have to be delineated with precision. Resonance enhancement of the Raman signals, when the incident or scattered photons approach excitonic transitions, provide an opportunity to extract the exchange contribution by comparing spin-flip Raman shifts in the DMS and those in the host. In the present study this approach has been successful for $\mathrm{Cd}_{1-x} \mathrm{~V}_{x}$ Te with $x$ as low as $4 \times 10^{-4}$. The $s-d$ exchange constant for $\mathrm{V}^{2+}$ deduced in this manner is $(285 \pm 8) \mathrm{meV}$. This value is comparable with those reported for the II-VI telluride, selenide, and sulfide based DMS's. ${ }^{46}$

The nature of the resonance enhancement, which emerged in the course of the investigation, exposed the role of the electronic levels of free and donor-bound excitons as intermediate levels in the spin-flip Raman process. In the specific case of free exciton mediated SFRS, the condition for in- and out-resonance is simultaneously fulfilled, with a resultant impressive enhancement, often referred to as double resonance.

\section{ACKNOWLEDGMENTS}

The authors thank the National Science Foundation for the support through Grant Nos. DMR-0102699 and ESC0129853 at Purdue University and through Grant No. DMR03-05653 and the Donors of the American Chemical Society Petroleum Research Fund PRF No. 40209-B5M at the University of North Florida.
${ }^{1}$ Diluted Magnetic Semiconductors, edited by J.K. Furdyna and J. Kossut, Semiconductors and Semimetals Vol. 25, edited by R.K. Willardson and A.C. Beer (Academic Press, San Diego, 1988).

${ }^{2}$ H. Munekata, H. Ohno, S. von Molnar, A. Segmüller, L.L. Chang, and L. Esaki, Phys. Rev. Lett. 63, 1849 (1989).

${ }^{3}$ F. Geist, W. Herbst, C. Mejia-Garcia, H. Pascher, R. Rupprecht, Y. Ueta, G. Springholz, G. Bauer, and M. Tacke, Phys. Rev. B 56, 13042 (1997); H. Krenn, W. Herbst, H. Pascher, Y. Ueta, G. Springholz, and G. Bauer, ibid. 60, 8117 (1999).

${ }^{4}$ T. Dietl, H. Ohno, F. Matsukura, J. Cibert, and D. Ferrand, Science 287, 1019 (2000); P. Kacman, Semicond. Sci. Technol. 16, R25 (2001).

${ }^{5}$ J. Blinowski, P. Kacman, and H. Przybylinska, Solid State Commun. 79, 1021 (1991); J. Blinowski and P. Kacman, Phys. Rev. B 46, 12298 (1992); J. Blinowski, P. Kacman, and J.A. Majewski, Acta Phys. Pol. A 88, 683 (1995); J. Blinowski, P. Kacman, and J.A. Majewski, J. Cryst. Growth 159, 972 (1996).

${ }^{6}$ W. Mac, Nguyen The Khoi, A. Twardowski, J.A. Gaj, and M. Demianiuk, Phys. Rev. Lett. 71, 2327 (1993); W. Mac, A. Twardowski, and M. Demianiuk, Phys. Rev. B 54, 5528 (1996); W. Mac, M. Herbich, A. Twardowski, and M. Demianiuk, Semicond. Sci. Technol. 15, 748 (2000).

${ }^{7}$ See, for example, A.K. Ramdas and S. Rodriguez, in Light Scattering in Solids, edited by M. Cardona and G. Güntherodt
(Springer, New York, 1991), Vol. VI, p. 137.

${ }^{8}$ D.L. Peterson, D.U. Bartholomew, U. Debska, A.K. Ramdas, and S. Rodriguez, Phys. Rev. B 32, 323 (1985).

${ }^{9}$ M.V. Alekseenko and A.I. Veinger, Sov. Phys. Semicond. 8, 143 (1974).

${ }^{10}$ M. Willatzen, M. Cardona, and N.E. Christensen, Phys. Rev. B 51, 17992 (1995).

${ }^{11}$ A. Nakamura, D. Paget, C. Hermann, C. Weisbuch, G. Lampel, and B.C. Cavenett, Solid State Commun. 30, 411 (1979).

${ }^{12}$ M. Oestreich, S. Hallstein, A.P. Heberle, K. Eberl, E. Bauser, and W.W. Rühle, Phys. Rev. B 53, 7911 (1996).

${ }^{13}$ P.E. Simmonds, H. Venghaus, R. Sooryakumar, and P.J. Dean, Solid State Commun. 43, 311 (1982).

${ }^{14}$ T.W. Walker, C.W. Litton, D.C. Reynolds, T.C. Collins, W.A. Wallace, J.H. Gorrell, and K.C. Jungling, in Proceedings of the XI International Conference on the Physics of Semiconductors, Warsaw, 1972, edited by M. Miasek (Elsevier, New York, 1972), p. 376.

${ }^{15}$ R.E. Slusher, C.K.N. Patel, and P.A. Fleury, Phys. Rev. Lett. 18, 77 (1967).

${ }^{16}$ F.F. Geyer and H.Y. Fan, IEEE J. Quantum Electron. QE-16, 1365 (1980).

${ }^{17}$ K. Cho, W. Dreybrodt, P. Hiesinger, S. Suga, and F. Willmann, in Proceedings of the XII International Conference on the Physics 
of Semiconductors, Stuttgart, 1974, edited by M.H. Pilkuhn (Teubner, Stuttgart, 1974), p. 945.

${ }^{18}$ D.G. Thomas and J.J. Hopfield, Phys. Rev. 175, 1021 (1968).

${ }^{19}$ J.M. Francou, K. Saminadayar, and J.L. Pautrat, Phys. Rev. B 41, 12035 (1990).

${ }^{20}$ W. Dreybrodt, K. Cho, S. Suga, F. Willmann, and Y. Niji, Phys. Rev. B 21, 4692 (1980).

${ }^{21}$ Ch. Neumann, A. Nöthe, and N.O. Lipari, Phys. Rev. B 37, 922 (1988)

${ }^{22}$ Y. Oka and M. Cardona, Phys. Rev. B 23, 4129 (1981).

${ }^{23}$ J.F. Scott, T.C. Damen, and P.A. Fleury, Phys. Rev. B 6, 3856 (1972).

${ }^{24}$ E. Molva and Le Si Dang, Phys. Rev. B 27, 6222 (1983); ibid. 32, 1156 (1985).

${ }^{25}$ D. Wolverson, P.J. Boyce, C.M. Townsley, B. Schlichtherle, and J.J. Davies, J. Cryst. Growth 159, 229 (1996).

${ }^{26}$ See, for example, A. Petrou, D.L. Peterson, S. Venugopalan, R.R. Galazka, A.K. Ramdas, and S. Rodriguez, Phys. Rev. B 27, 3471 (1983); J. Stühler, M. Hirsch, G. Schaack, and A. Waag, ibid. 49, 7345 (1994).

${ }^{27}$ R. Loudon, Adv. Phys. 13, 423 (1964).

${ }^{28}$ A. Jayaraman, G.A. Kourouklis, R. People, S.K. Sputz, and L. Pfeiffer, Pramana, J. Phys. 35, 167 (1990).

${ }^{29}$ S.I. Gubarev, T. Ruf, and M. Cardona, Phys. Rev. B 43, 1551 (1991).

${ }^{30}$ S.I. Gubarev, T. Ruf, and M. Cardona, Phys. Rev. B 43, 14564 (1991)

${ }^{31}$ J. Kreissl and H.-J. Schulz, J. Cryst. Growth 161, 239 (1996).

${ }^{32}$ For the description of the notation see, for instance, A.M. Hennel, in Semiconductors and Semimetals, edited by E.R. Weber (Academic Press, New York, 1993), Vol 38, p. 189.
${ }^{33}$ J.M. Baranowski, J.M. Langer, and S. Stefanova, in Proceedings of the XI International Conference on the Physics of Semiconductors, Warsaw, 1972, edited by M. Miasek (Elsevier, New York, 1972), p. 1001.

${ }^{34}$ P. Peka, M.U. Lehr, H.-J. Schulz, R. Schwarz, and K.W. Benz, Appl. Phys. A: Solids Surf. 58, 447 (1994).

${ }^{35}$ P. Christmann, J. Kreissl, D.M. Hofmann, B.K. Meyer, R. Schwarz, and K.W. Benz, J. Cryst. Growth 161, 259 (1996).

${ }^{36}$ H.R. Selber, P. Peka, S.W. Biernacki, H.-J. Schulz, R. Schwarz, and K.W. Benz, Semicond. Sci. Technol. 14, 521 (1999).

${ }^{37}$ P. Rudolph, Prog. Cryst. Growth Charact. 29, 275 (1994).

${ }^{38}$ H.J. von Bardeleben, J.C. Launay, and V. Mazoyer, Appl. Phys. Lett. 63, 1140 (1993).

${ }^{39}$ R.N. Schwartz, M. Ziari, and S. Trivedi, Phys. Rev. B 49, 5274 (1994).

${ }^{40}$ P.A. Slodowy and J.M. Baranowski, Phys. Status Solidi B 49, 499 (1972).

${ }^{41}$ M. Villeret, S. Rodriguez, and E. Kartheuser, Phys. Rev. B 41, 10 028 (1990).

${ }^{42}$ See, for instance, T.M. Pekarek, J.E. Luning, I. Miotkowski, and B.C. Crooker, Phys. Rev. B 50, 16914 (1994).

${ }^{43}$ T.M. Pekarek, I. Miotkowski, and B.C. Crooker, J. Appl. Phys. 79, 6436 (1996).

${ }^{44}$ J. Kossut, Phys. Status Solidi B 78, 537 (1976).

${ }^{45}$ See, for instance, H. Alawadhi, I. Miotkowski, V. Souw, M. McElfresh, A.K. Ramdas, and S. Miotkowska, Phys. Rev. B 63, 155201 (2001).

${ }^{46}$ D. Heiman, in Proceedings of the 12th International Conference on High Magnetic Fields in the Physics of Semiconductors II, Vol. 2, Würzburg, 1996, edited by G. Landwehr and W. Ossau (World Scientific, 1997), p. 847. 